\title{
Ejemplo de buena práctica de evaluación formativa y compartida en la formación inicial del profesorado de educación infantil
}

\section{A good practice of Formative and Shared Assessment in pre-service Teacher Education in Childhood Education}

\author{
Miriam Molina Soria ${ }^{1}$, Víctor M. López Pastor ${ }^{2}$, Cristina Pascual Arias ${ }^{3}$ \& Raúl Alberto \\ Barba Martín ${ }^{4}$
}

Fecha de recepción: 24/01/2019; Fecha de revisión: 22/02/2019; Fecha de aceptación: 25/03/2019

Cómo citar este artículo:

Molina, M., López-Pastor, V. V., Pascual, C., \& Barba, R. (2019). Ejemplo de buena práctica de evaluación formativa y compartida en la formación inicial del profesorado de educación infantil. Revista de Innovación y Buenas Prácticas Docentes, 8, 69-86.

Autor de Correspondencia: Víctor M. López Pastor, vlopez@mpc.uva.es

\section{Resumen:}

En este trabajo presentamos los resultados de una buena práctica llevada a cabo en la asignatura de Fundamentos de la educación física en educación infantil: los "Proyectos de Aprendizaje tutorado". En la primera parte se describe la organización de la asignatura y el ejemplo de buena práctica. En la segunda parte se presentan los resultados obtenidos, que son muy positivos. Hay un cambio relevante en la carga de trabajo del profesor, que puede explicarse por los años de experiencia del profesorado que desarrolla la asignatura. Se refleja en conclusiones.

Palabras clave: Educación Física, Educación Infantil, Proyecto de Trabajo Tutorado, Evaluación Formativa, Evaluación Compartida.

\section{Abstract:}

In this work we present the results of a good practice carried out in the subject of Foundations of Physical Education in Early Childhood Education: the "Tutored Learning Projects". The first part describes the organization of the subject and the example of good practice. In the second part the results obtained are presented, which are very positive. There is a relevant change in the workload of the professor, which can be explained by the years of experience of the teaching staff that develops the subject. It is reflected in conclusions.

Key Words: Physical Education, Early Childhood Education, Tutored Learning Projects, Formative Assessment, Shared Assessment.

\footnotetext{
1 Universidad de Valladolid (España), miriam.molina @uva.es; CÓDIGO ORCID: 0000-0003-2974-5535

2 Universidad de Valladolid (España), vlopez@mpc.uva.es; CÓDIGO ORCID: 0000-0003-2681-9543

3 Universidad de Valladolid (España), cristina.pascual@uva.es; CÓDIG O ORCID: 0000-0002-2781-5600

${ }^{4}$ Universidad de Valladolid (España), ra ula lberto.brba@uva.es; CÓ DIG O ORCID: https://orcid.org/0000-0003-0071-687X
} 


\section{INTRODUCCIÓN}

En la Facultad de Educación de Segovia llevamos muchos años trabajando con los Proyectos de Aprendizaje Tutorado (PAT), porque han demostrado ser una forma eficaz de trabajo teóricopráctico y de desarrollo de competencias profesionales (Barba, López, Manrique, Gea, y Monjas, 2010; Barba-Martín y López-Pastor, 2017; López, Manrique, Monjas y Gea, 2010; Manrique, López, Monjas, y Real, 2010). Se trata de una actividad de aprendizaje grupal. Cada grupo, de entre 2 a 5 alumnos, tiene que preparar una sesión práctica y un marco teórico a elegir dentro de una lista de temas y propuestas de intervención que se ofrecen a la clase a principio de curso. Se asigna una fecha de realización para cada PAT. En tutorías se explica a cada grupo los documentos que tienen que leer sobre cada propuesta y deben realizar una serie de tutorías obligatorias con los borradores de sus PATs antes de poder llevarlo a cabo, de forma que se asegure una calidad mínimamente aceptable en la puesta en práctica y en la documentación a entregar a sus compañeras. En muchas asignaturas el PAT se lleva a cabo con alumnado de infantil o primaria, en horario lectivo real, en algunos de los centros educativos de la ciudad.

Los PAT han demostrado ser una actividad de aprendizaje más interesante para el alumnado, pero mucho más complejo de organizar para el profesorado. Esta complejidad aumenta cuando el número de alumnos y grupos es tan elevado como en nuestra experiencia. El profesorado no solo se encarga de tutorizar todos los PAT, sino que debe estar atento a las cuestiones de logística y organización de cada uno de los trabajos. Teniendo que preparar previamente un cronograma donde asegure que todos los PAT dispondrán de un espacio y tiempo adecuado para su puesta en práctica. Además, de proveer de todos los materiales necesarios, siempre que sea posible, a cada grupo. Estos aspectos organizativos se complican aún más cuando la puesta en práctica se realiza en los centros educativos con el alumnado de infantil o primaria. Pues el profesorado debe coordinar un cronograma que satisfaga las posibilidades del maestro que presta su aula, del alumnado y las suyas propias para asistir a la puesta en práctica.

Los procesos de evaluación durante el PAT son la principal actividad de aprendizaje. En nuestra asignatura (Fundamentos de la Educación Física en Infantil) y en el PAT utilizamos una evaluación formativa y compartida que centra su finalidad en conseguir información para mejorar los procesos de enseñanza-aprendizaje tanto para el alumnado como en lo relativo a la práctica docente (López-Pastor, 2006; López-Pastor et al. 2008). Es una forma de evaluación continua y planificada para trata de ayudar al alumnado a conseguir un aprendizaje de calidad y más satisfactorio a través de continuos feedbacks (Biggs, 2005; Brown \& Glasner, 2003; Hamodi, Moreno-Murcia, \& Barba-Martín, 2018).

En este artículo analizaremos la puesta en práctica del PAT en el año 2018 en nuestra asignatura, mostrando los principales resultados en el aprendizaje y valoración del alumnado hacia la práctica y el sistema de evaluación.

\section{DESARROLLO DE LA EXPERIENCIA DE INNOVACIÓN}

Durante el curso académico 2017/2018 llevamos a cabo PATs en todas las asignaturas que impartimos. Aunque, la forma de actuación, en rasgos generales, es igual para cada una de ellas. Las especificidades de la asignatura y el alumnado hacen que en algunos momentos adaptemos nuestra práctica. Por ello, a continuación analizaremos cuáles son los principales puntos en el desarrollo de nuestros PAT en el contexto específico que planteamos. 


\subsection{Contexto}

Nuestra experiencia tiene lugar en la Facultad de Educación de Segovia perteneciente a la Universidad de Valladolid. La asignatura en la que se ha realizado la buena práctica del PAT ha sido Fundamentos y didáctica de la educación corporal en Educación Infantil, dentro del tercer curso del título universitario de grado de maestro en Educación Infantil. En la Figura 1 podemos ver los detalles de esta asignatura.

\begin{tabular}{lc}
\hline \multicolumn{2}{c}{ Características de la asignatura } \\
\hline Tipo de asignatura & Cuatrimestral/Semestral \\
& Créditos Totales: 6 \\
& Créditos presenciales: 2,4 \\
Créditos ECTS totales de docencia del profesor & $\bullet$ Gran grupo: 1,2 \\
y su tipología & $\bullet$ Grupos reducidos: 1,2 \\
& $\bullet$ Grupos de prácticas: 0 \\
& Créditos no presenciales: 3,6 \\
\hline
\end{tabular}

Figura 1. Contexto de aplicación de la experiencia.

La asignatura contó con un total de 43 alumnos y alumnas. El alumnado se dividía, excepto para las clases prácticas, en 2 grupos, contando con 21 alumnos en el grupo A y 22 alumnos en el grupo B.

\subsubsection{Sistema de evaluación, actividades y calificación de la asignatura}

El alumnado debe realizar una serie de actividades de aprendizaje a lo largo de la asignatura, entre ellas se encuentra el PAT. Todas estas actividades son evaluadas por el profesorado y el alumnado a través de diferentes instrumentos como escalas descripticas o plantillas de corrección. Pudiendo así, el profesor, proporcionar continuos feedback al alumnado. La distribución del peso en la calificación de cada actividad de aprendizaje se realiza en función del trabajo y dedicación que cada uno de ellos supondrá al alumnado. Estos porcentajes son compartidos y debatidos con el alumnado a principio de curso, pudiendo modificarse. Este año, tras debatirlos con el alumnado, quedaron de la siguiente forma (Tabla 1).

Tabla 1.

Resumen de las actividades de evaluación y calificación de la asignatura.

\begin{tabular}{llc}
\hline \multicolumn{1}{c}{ Actividades de aprendizaje } & \multicolumn{1}{c}{ EVALUACIÓN } & $\begin{array}{c}\text { Calificación } \\
\%\end{array}$ \\
\hline 1. PAT & - Escala descriptiva (rúbrica) & 30 \\
2. Trabajo grupal sobre el currículum de El y recensiones & - Escala descriptiva & 20 \\
sobre lecturas & & 20 \\
3. Fichas de sesiones prácticas & - Escala descriptiva (rúbrica) & 20 \\
4. Examen parcial con co-evaluación inmediata & - Plantilla de corrección "ad hoc" & 10 \\
5. Mapas conceptuales & - Escala descriptiva (rúbrica) & \\
\hline
\end{tabular}

Si bien, el profesorado ofertamos las anteriores actividades de aprendizaje para el alumnado, no todas ellas son obligatorias, únicamente lo son el PAT y el examen. El alumnado, en función de sus necesidades o intereses puede elegir cursar la asignatura a través de tres vías: (a) una primera vía continua, donde el alumnado debe cumplir una serie de requisitos que son, la asistencia obligatoria, pudiendo faltar únicamente un $20 \%$ y siempre de forma justificada y la entrega de todas las actividades de aprendizaje en los tiempos marcados. En esta vía se siguen los porcentajes de evaluación marcados anteriormente; (b) una segunda vía mixta, mediante la cual el alumnado solo tiene la obligación de realizar el PAT y el examen, todos las demás actividades de aprendizaje serán de carácter voluntario. En este caso el alumnado debe asistir, al menos, al $50 \%$ de las clases. Sus 
porcentajes de calificación varían, siendo un 30\% para el PAT, un 50\% para el examen y un 20\% para los trabajos voluntarios y; (c) una tercera vía final, donde el alumnado no tiene ningún compromiso de asistencia a la asignatura. Debe realizar el PAT y llevarlo a cabo en un centro. De esta forma el día del examen realizará un examen final teórico, un examen práctico y la exposición del marco teórico y puesta en práctica de su PAT.

\subsection{Diseño y puesta en práctica del PAT}

La realización del PAT tiene una serie de pasos que el alumnado debe ir siguiendo y cuya finalidad es pode dotar de espacio y tiempo a cada grupo, así como de calidad en los trabajos que se realicen. El proceso de elaboración es el siguiente:

1. Se asigna una fecha de realización para cada PAT.

2. En tutorías se explica a cada grupo los documentos que tienen que leer sobre cada propuesta.

3. El grupo elabora un borrador del marco teórico (4 páginas) y un plan de sesión y acuden a una tutoría grupal, donde el profesor corrige los borradores y les dice los aspectos que deben ser mejorados y corregidos.

4. Este proceso se repite hasta que los dos documentos tienen una calidad aceptable.

5. Se realiza la sesión práctica en la fecha elegida y a continuación se expone el marco teórico, de forma oral, en 10 minutos, entregando previamente una copia en papel del mismo a cada compañero.

6. Una semana después deben entregar el informe final del PAT. Si está correcto el profesor se lo devuelve para que lo guarden en su carpeta. Si hay aspectos a mejorar deben corregirlo y volverlo a entregar en un plazo máximo de una semana.

Durante todo este proceso, el alumnado cuenta con una serie de instrumentos de evaluación para poder controlar la calidad de su trabajo y su aprendizaje. Dichos instrumentos son entregados al alumnado antes de comenzar la elaboración del PAT. De esta forma, intentamos establecer de forma clara el procedimiento a seguir en el PAT y en el proceso de evaluación. Los instrumentos de evaluación son trabajados por el alumnado, junto con el profesorado, en las tutorías y las correcciones, proporcionándose feedbacks a través de ellos durante todo el proceso de elaboración. El grupo tiene la obligatoriedad de asistir a todas las tutorías que sean necesarias para garantizar una calidad mínima del PAT antes de llevarlo a cabo. Los instrumentos que utilizamos en nuestra experiencia se pueden observar en la siguiente figura.

\section{Instrumentos de evaluación}

Fichas de autoevaluación para el PAT

Escalas descriptiva para la evaluación y la calificación del PAT

Rúbrica específica de autoevaluación del PAT

Ficha de observación de la presentación oral del PAT del grupo y de cada componente.

Figura 2. Instrumentos de evaluación utilizados durante el PAT. 


\section{RESULTADOS}

A continuación mostramos los principales resultados que extrajimos mediante el cuestionario elaborado por la Red de Evaluación Formativa y Compartida y que fue realizado por nuestro alumnado al finalizar la asignatura. A través de las respuestas, de forma anónima, al cuestionario analizaremos la valoración general que el alumnado otorga a la experiencia del PAT, las principales ventajas e inconvenientes del proceso de evaluación seguido durante el PAT y la auto-percepción que tiene el alumnado de las competencias adquiridas.

Además, se incluyen los resultados de rendimiento académico de la asignatura y la carga de trabajo del profesorado, al ser factores importantes a tener en cuenta en el desarrollo de la experiencia.

\subsection{Valoración de la experiencia del PAT}

Los resultados en torno a la valoración de la experiencia los presentamos en las tablas 2 y 3 :

Tabla 2. Cuestiones preguntadas al alumnado sobre la experiencia.

\begin{tabular}{|c|c|c|c|c|c|c|}
\hline \multirow{2}{*}{$\begin{array}{l}\text { Has realizado alguna vez esta experiencia } \\
\text { en otra asignatura } \\
\text { Items del cuestionario }\end{array}$} & \multicolumn{2}{|c|}{ SI (64.32 \%) } & \multicolumn{3}{|c|}{ No (35.68 \%) } & \multirow{2}{*}{$\begin{array}{l}1,36 \\
\text { MEDIA } \\
(1-5)\end{array}$} \\
\hline & Nada & Poco & Algo & Bastante & Mucho & \\
\hline $\begin{array}{l}\text { 1¿Se ha negociado la utilización de esta } \\
\text { experiencia en la asignatura al comienzo } \\
\text { del curso }\end{array}$ & & & $x$ & & & 3.16 \\
\hline $\begin{array}{l}2 i \text { Crees que esta experiencia te ha } \\
\text { ayudado a adquirir competencias } \\
\text { profesionales? }\end{array}$ & & & & & $x$ & 4.34 \\
\hline $\begin{array}{l}3 \text { ¿La evaluación que se ha planteado } \\
\text { favorece la adquisición de las } \\
\text { competencias profesionales }\end{array}$ & & & & $X$ & & 4 \\
\hline $\begin{array}{l}4 ¿ \text { Consideras útil lo aprendido con esta } \\
\text { experiencia? }\end{array}$ & & & & & $x$ & 4.38 \\
\hline $\begin{array}{l}5 \text { ¿Qué es lo más útil que has aprendido? } \\
\text { Que es una experiencia:... }\end{array}$ & & & & & & \\
\hline $\begin{array}{l}\text { 5.1. innovadora, porque desarrolla } \\
\text { soluciones nuevas o creativas }\end{array}$ & & & & $x$ & & 3.69 \\
\hline $\begin{array}{l}\text { 5.2. efectiva, porque demuestra un } \\
\text { impacto positivo y tangible de mejora }\end{array}$ & & & & $x$ & & 4.03 \\
\hline $\begin{array}{l}\text { 5.3. sostenible, porque se mantiene en el } \\
\text { tiempo y puede producir efectos } \\
\text { duraderos }\end{array}$ & & & & $x$ & & 4.06 \\
\hline $\begin{array}{l}\text { 5.4. replicable, cuando es posible utilizarla } \\
\text { como modelo para desarrollarla en otros } \\
\text { contextos }\end{array}$ & & & & $x$ & & 4.25 \\
\hline
\end{tabular}


Tabla 2. Cuestiones preguntadas al alumnado sobre la experiencia (continuación).

\begin{tabular}{|c|c|c|c|c|c|c|}
\hline Items del cuestionario & Nada & Poco & Algo & Bastante & Mucho & $\begin{array}{l}\text { MEDIA } \\
(1-5)\end{array}$ \\
\hline & $\begin{array}{l}\text { Muy } \\
\text { insufi- } \\
\text { ciente. }\end{array}$ & $\begin{array}{l}\text { Insufi- } \\
\text { ciente }\end{array}$ & $\begin{array}{l}\text { Sufi- } \\
\text { ciente }\end{array}$ & Buenas & $\begin{array}{l}\text { Muy } \\
\text { Buenas }\end{array}$ & \\
\hline $\begin{array}{l}6 \text { ¿Cómo valoras las ayudas recibidas por } \\
\text { el docente? }\end{array}$ & & & & & $x$ & 4.3 \\
\hline $\begin{array}{l}7 \text { ¿Cómo valoras las ayudas recibidas por } \\
\text { los compañeros? }\end{array}$ & & & & $x$ & & 3.84 \\
\hline & $\begin{array}{l}\text { Nada } \\
\text { satisfe. }\end{array}$ & $\begin{array}{l}\text { Poco } \\
\text { satisfe }\end{array}$ & $\begin{array}{l}\text { Satis- } \\
\text { facción } \\
\text { media. }\end{array}$ & $\begin{array}{l}\text { Bast. } \\
\text { Satisfe. }\end{array}$ & $\begin{array}{l}\text { Muy } \\
\text { satisfe }\end{array}$ & $\begin{array}{c}\text { MEDI } \\
\text { A } \\
(1-5)\end{array}$ \\
\hline $\begin{array}{l}8 \text { Señala la satisfacción global en relación } \\
\text { con la experiencia }\end{array}$ & & & & $x$ & & 4.09 \\
\hline $\begin{array}{l}9 \text { Señala la satisfacción global en relación } \\
\text { con la evaluación de la experiencia }\end{array}$ & & & & $x$ & & 3.84 \\
\hline $\begin{array}{l}10 \text { ¿Cuál es el grado de dificultad de la } \\
\text { experiencia? }\end{array}$ & & & $x$ & & & 3.44 \\
\hline
\end{tabular}

Tabla 3. Valoración de la experiencia de buena práctica como docente que la lleva a cabo.

\begin{tabular}{|c|c|c|c|}
\hline Indicador & prof & Valoración Q profe (¿Por qué?) & $\begin{array}{c}\text { Valoración } \\
\text { alumnos } \\
(1-5)\end{array}$ \\
\hline $\begin{array}{l}\text { (a) innovadora, al } \\
\text { desarrollar soluciones } \\
\text { nuevas o creativas }\end{array}$ & Si $x$ & $\begin{array}{l}\text { Porque supone una gran diferencia con } \\
\text { modelos más tradicionales de enseñanza. } \\
\text { Es una metodología activa y exige una } \\
\text { fuerte colaboración grupal para que salga } \\
\text { bien. }\end{array}$ & 4.69 \\
\hline $\begin{array}{l}\text { (b) efectiva, cuando } \\
\text { demuestra un impacto } \\
\text { positivo y tangible de } \\
\text { mejora }\end{array}$ & Si $\mathrm{x}$ & $\begin{array}{l}\text { Porque al ser obligatoria la realización de } \\
\text { tutorías durante la elaboración del } \\
\text { proyecto, la calidad del mismo mejora } \\
\text { considerablemente. }\end{array}$ & 4.03 \\
\hline $\begin{array}{l}\text { (c) sostenible, al } \\
\text { mantenerse en el } \\
\text { tiempo y producir } \\
\text { efectos duraderos }\end{array}$ & Six & $\begin{array}{l}\text { Porque supone una mayor carga de trabajo } \\
\text { en las tutorías de los meses centrales, } \\
\text { cuando la mayoría de los grupos están } \\
\text { elaborando su PAT y requieren tutorías, } \\
\text { pero es perfectamente viable, dado que se } \\
\text { realiza en las horas oficiales de tutoría }\end{array}$ & 4.06 \\
\hline $\begin{array}{l}\text { (d) replicable, cuando } \\
\text { es posible utilizarla } \\
\text { como modelo para } \\
\text { desarrollarla en otros } \\
\text { contextos }\end{array}$ & Six & $\begin{array}{l}\text { Porque se puede utilizar en cualquier } \\
\text { asignatura. De hecho, en nuestro centro es } \\
\text { una metodología muy utilizada desde hace } \\
\text { 15-20 años en muchas asignaturas. }\end{array}$ & 4.25 \\
\hline
\end{tabular}

Como podemos observar en los resultados presentados a través de la tabla 2 y 3 , el PAT se trata de una actividad de aprendizaje que funciona muy bien, con una tasa de éxito muy elevada y con una buena calidad general. La explicación de ello está en la obligatoriedad y sistematicidad del proceso de revisión antes de poder poner en práctica el PAT. A pesar de ello este año ha habido problemas con un grupo que lo llevaba muy mal preparado y tarde. Esta situación resulta 
complicada evitarla del todo, a pesar de las tutorías grupales. Pero son pocos casos, la mayoría funcionó muy bien.

Además, en el alumnado destaca como resultado importante el alto porcentaje sobre el aprendizaje generado con la experiencia. Sin embargo, como desventajas el alumnado también destaca la descoordinación, por momentos, de la asignatura. Aspecto que se explica al haber realizado la asignatura este curso entre 4 maestros noveles. También piden reducir la carga de trabajo y que el profesorado ofrezca otras alternativas además de las tres vías de calificación ya que ven la obligatoriedad de asistencia difícilmente justificable en algunas situaciones.

Como propósito para el siguiente curso trataremos de utilizar de forma sistemática la plantilla de autoevaluación y autocalificación del PAT que ya se empleo el año anterior. Tratando así de ofrecer una orientación importante al alumnado.

\subsection{Aspectos en relación a la asignatura a nivel general}

Como hemos venido describiendo, el proceso de evaluación del PAT y de la asignatura en general es uno de los procesos de aprendizaje más importante en nuestra experiencia. Por ello, a continuación analizaremos las principales ventajas e inconvenientes del sistema de evaluación de la asignatura y el PAT. Además, analizaremos la auto-percepción que el alumnado tiene del aprendizaje conseguido (ver figuras 3 y 4 ).

\begin{tabular}{|c|c|c|c|c|}
\hline $\begin{array}{l}\text { Aspectos que se consideran por parte del } \\
\text { ALUMNADO }\end{array}$ & Nada & Algo & Bastante & Mucho \\
\hline 1. Ofrece alternativas a todos los estudiantes & & & & $\mathrm{x}$ \\
\hline $\begin{array}{l}\text { 2. Hay un contrato previo, negociado y } \\
\text { consensuado del sistema de evaluación }\end{array}$ & & $\mathrm{x}$ & & \\
\hline $\begin{array}{l}\text { 3. Está centrado en el proceso, importancia del } \\
\text { trabajo diario }\end{array}$ & & & & $\mathrm{x}$ \\
\hline 4. El estudiante realiza un aprendizaje activo & & & & $x$ \\
\hline $\begin{array}{l}\text { 5. Se plantea el trabajo en equipo de forma } \\
\text { colaborativa }\end{array}$ & & & & $\mathrm{x}$ \\
\hline $\begin{array}{l}\text { 6. El alumno/a está más motivado, el proceso de } \\
\text { aprendizaje es más motivador }\end{array}$ & & & $\mathrm{x}$ & \\
\hline 7. La calificación es más justa & & & $\mathrm{x}$ & \\
\hline $\begin{array}{l}\text { 8. Mejora la tutela académica (seguimiento y ayuda } \\
\text { al alumno/a) }\end{array}$ & & & $\mathrm{x}$ & \\
\hline 9. Ofrece alternativas a todos los estudiantes & & & & $x$ \\
\hline $\begin{array}{l}\text { 10. Hay un contrato previo, negociado y } \\
\text { consensuado del sistema de evaluación }\end{array}$ & & $x$ & & \\
\hline $\begin{array}{l}\text { 11. Está centrado en el proceso, importancia del } \\
\text { trabajo diario }\end{array}$ & & & & $x$ \\
\hline 12. El estudiante realiza un aprendizaje activo & & & & $x$ \\
\hline $\begin{array}{l}\text { 13. Se plantea el trabajo en equipo de forma } \\
\text { colaborativa }\end{array}$ & & & & $\mathrm{x}$ \\
\hline $\begin{array}{l}\text { 14. El alumno/a está más motivado, el proceso de } \\
\text { aprendizaje es más motivador }\end{array}$ & & & $\mathrm{x}$ & \\
\hline 15. La calificación es más justa & & & $\mathrm{x}$ & \\
\hline $\begin{array}{l}\text { 16. Mejora la tutela académica (seguimiento y } \\
\text { ayuda al alumno/a) }\end{array}$ & & & $\mathrm{x}$ & \\
\hline 17. Permite aprendizajes funcionales & & & $x$ & \\
\hline 18. Genera aprendizajes significativos & & & & $x$ \\
\hline
\end{tabular}

Figura 3. Ventajas que se reconocen en el sistema de evaluación aplicado. 


\begin{tabular}{|c|c|c|c|c|c|}
\hline & Nada & Poco & Algo & Bastante & Mucho \\
\hline 19. Se aprende mucho más & & & & $\mathrm{x}$ & $\mathrm{x}$ \\
\hline 20. Mejora la calidad de los trabajos exigidos & & & & $x$ & \\
\hline 21. Hay interrelación entre teoría y práctica & & & & & $x$ \\
\hline $\begin{array}{l}\text { 22. Evalúa todos los aspectos posibles (en } \\
\text { referencia al saber, saber hacer y saber estar y } \\
\text { ser) }\end{array}$ & & & & $\mathrm{x}$ & \\
\hline $\begin{array}{l}\text { 23. Hay retroalimentación en documentos y } \\
\text { actividades }\end{array}$ & & & & & $\mathrm{x}$ \\
\hline $\begin{array}{l}\text { 24. Hay posibilidad de corregir errores en } \\
\text { documentos y actividades }\end{array}$ & & & & & $\mathrm{x}$ \\
\hline 25. Se da un seguimiento más individualizado & & & & $x$ & \\
\hline 26. Requiere más responsabilidad & & & & & $\mathrm{x}$ \\
\hline $\begin{array}{l}\text { Aspectos que se consideran por parte del } \\
\text { PROFESORADO }\end{array}$ & Nada & Poco & Algo & Bastante & Mucho \\
\hline $\begin{array}{l}\text {-Satisfacción del profesor con el sistema de } \\
\text { evaluación utilizado }\end{array}$ & & & & & $\mathrm{x}$ \\
\hline
\end{tabular}

Figura 3. Ventajas que se reconocen en el sistema de evaluación aplicado (continuación).

\begin{tabular}{|c|c|c|c|c|c|}
\hline Aspectos que se consideran por parte del alumnado & Nada & Poco & Algo & Bastante & Mucho \\
\hline 1. Exige una asistencia obligatoria y activa & & & & & $\mathrm{x}$ \\
\hline $\begin{array}{l}\text { 2. Tiene una dinámica de trabajo poco conocida, falta de } \\
\text { hábito }\end{array}$ & & & & $\mathrm{x}$ & \\
\hline 3. Exige continuidad & & & & & $\mathrm{x}$ \\
\hline 4. Hay que comprenderlo previamente & & & $\mathrm{x}$ & & \\
\hline 5. Exige un mayor esfuerzo & & & & & $\mathrm{x}$ \\
\hline 6. Existe dificultad para trabajar en grupo & $\mathrm{x}$ & & & & \\
\hline 7. Se puede acumular mucho trabajo al final & & & $\mathrm{x}$ & & \\
\hline 8. Existe una desproporción trabajo/créditos & & & & & $\mathrm{x}$ \\
\hline $\begin{array}{l}\text { 9. El proceso de calificación es más complejo y, a veces, } \\
\text { poco claro }\end{array}$ & & & $\mathrm{x}$ & & \\
\hline $\begin{array}{l}\text { 10. Genera inseguridad e incertidumbre, dudas sobre } \\
\text { que hay que realizar }\end{array}$ & & & $\mathrm{x}$ & & \\
\hline 11. Es injusto frente a otros procesos de evaluación & $\mathrm{x}$ & & & & \\
\hline 12. Las correcciones han sido poco claras & $\mathrm{x}$ & & & & \\
\hline 13. La valoración del trabajo es subjetiva & $\mathrm{x}$ & $\mathrm{x}$ & & & \\
\hline $\begin{array}{l}\text { 14. Exige participar en mi propia evaluación } \\
\text { (autoevaluarse) }\end{array}$ & & & & $\mathrm{x}$ & \\
\hline $\begin{array}{l}\text { Aspectos que se consideran por parte del } \\
\text { PROFESORADO }\end{array}$ & Nada & Poco & Algo & Bastante & Mucho \\
\hline $\begin{array}{l}\text { - Supone una carga de trabajo excesiva para el } \\
\text { profesor }\end{array}$ & & & & $x$ & \\
\hline $\begin{array}{l}\text { - El alumnado presenta fuertes resistencias frente al } \\
\text { sistema de evaluación continua y formativa }\end{array}$ & & & $x$ & & \\
\hline
\end{tabular}

Figura 4. Inconvenientes que se reconocen en el sistema de evaluación aplicado. 
En cuanto a los datos de auto-percepción de competencias del alumnado, estos se dividieron en tres bloques. El primer bloque de competencias transversales, el segundo bloque de competencias docentes y el tercer bloque de competencias docentes específicas de Educación Física. Los resultados fueron los presentados en las tablas 4, 5 y 6.

Tabla 4.

Datos del cuestionario de autopercepción de competencias. Bloque I

\begin{tabular}{|c|c|c|c|c|}
\hline \multicolumn{5}{|c|}{ Bloque I: Competencias transversales } \\
\hline \multirow[b]{2}{*}{ ITEMS } & \multicolumn{2}{|c|}{$\begin{array}{l}\text { Principio } \\
\text { asignatura }\end{array}$} & \multicolumn{2}{|c|}{ Final Asignatura } \\
\hline & Media & DT & Media & DT \\
\hline 1.1. Analizar y sintetizar & 3 & .378 & 3.32 & .541 \\
\hline 1.2. Organizar y planificar & 3.28 & .591 & 3.52 & .508 \\
\hline 1.3. Comunicarse de forma oral y escrita en lengua nativa & 3.21 & .620 & 3.35 & .551 \\
\hline 1.4. Comunicarse gestual y corporalmente & 3.11 & .737 & 3.52 & .570 \\
\hline 1.5. Conocer una lengua extranjera & 2.48 & .643 & 2.23 & .679 \\
\hline $\begin{array}{l}\text { 1.6. Utilizar las Tecnologías de la Información y la Comunicación } \\
\text { en el ámbito de estudio }\end{array}$ & 3.11 & .737 & 3.13 & .718 \\
\hline 1.7. Trabajar en equipo & 3.59 & .568 & 3.87 & .341 \\
\hline $\begin{array}{l}\text { 1.8. Desarrollar habilidades en las relaciones interpersonales } \\
\text { (empatía, asertividad, respeto y escucha) }\end{array}$ & 3.72 & .528 & 3.84 & .374 \\
\hline $\begin{array}{l}\text { 1.9. Desarrollar habilidades intrapersonales (autoestima, } \\
\text { motivación y confianza en uno mismo) }\end{array}$ & 3.32 & .548 & 3.67 & .479 \\
\hline 1.10. Razonar de forma crítica y reflexiva & 2.97 & .626 & 3.19 & .601 \\
\hline 1.11. Desarrollar un compromiso ético & 3.10 & .673 & 3.48 & .570 \\
\hline 1.12. Desarrollar un aprendizaje autónomo & 3.34 & .614 & 3.43 & .626 \\
\hline 1.13. Adaptarse a situaciones nuevas & 3.34 & .553 & 3.42 & .672 \\
\hline 1.14. Desarrollar la creatividad & 3.10 & .772 & 3.45 & .624 \\
\hline
\end{tabular}

Tabla 5.

Datos de cuestionario de autopercepción de competencias. Bloque II.

\begin{tabular}{lcccc}
\hline \multicolumn{1}{c}{ Bloque II: Competencias docentes } & & & \\
& \multicolumn{2}{c}{$\begin{array}{c}\text { Principio } \\
\text { asignatura }\end{array}$} & \multicolumn{2}{c}{ Final } \\
& Medianatura \\
& DT & Media & DT \\
\hline 2.1. Conocer las características organizativas de los centros & 2.68 & .548 & 2.90 & .473 \\
educativos & & & & \\
2.2. Elaborar propuestas de cambio de la realidad educativa & 2.76 & .636 & 3.03 & .657 \\
2.3. Diseñar situaciones de aprendizaje & 3.21 & .412 & 3.42 & .502 \\
2.4. Animar situaciones de aprendizaje & 3.29 & .460 & 3.42 & .620 \\
2.5. Gestionar la progresión de los aprendizajes & 2.79 & .491 & 3.19 & .654 \\
2.6. Diseñar estrategias de atención a la diversidad & 2.79 & .491 & 3.10 & .662 \\
2.7. Poner en práctica estrategias de atención a la diversidad & 2.66 & .484 & 2.83 & .699 \\
2.8. Implicar al alumnado en su aprendizaje & 3.31 & .604 & 3.29 & .693 \\
2.9. Implicar al alumnado en la vida del centro & 3.03 & .778 & 2.97 & .605 \\
\hline
\end{tabular}


Tabla 5.

Datos de cuestionario de autopercepción de competencias. Bloque II (continuación).

\begin{tabular}{|c|c|c|c|c|}
\hline \multicolumn{5}{|c|}{ Bloque II: Competencias docentes } \\
\hline & \multicolumn{2}{|c|}{$\begin{array}{l}\text { Principio } \\
\text { asignatura }\end{array}$} & \multicolumn{2}{|c|}{$\begin{array}{c}\text { Final } \\
\text { Asignatura }\end{array}$} \\
\hline & Media & DT & Media & DT \\
\hline 2.10. Participar en la gestión del Centro & 2.21 & .774 & 2.39 & .761 \\
\hline 2.11. Informar a las familias sobre la evolución de su hijo/a & 2.61 & .629 & 2.71 & .783 \\
\hline 2.12. Implicar a las familias en el aprendizaje de su hijo/a & 2.85 & .718 & 3.03 & .657 \\
\hline 2.13. Implicar a las familias en la vida del centro & 2.89 & .629 & 2.74 & .773 \\
\hline 2.14. Afrontar los deberes y dilemas éticos de la profesión & 2.86 & .525 & 3.10 & .700 \\
\hline $\begin{array}{l}\text { 2.15. Atender al propio desarrollo profesional docente } \\
\text { (autoevaluación, lecturas, cursos, jornadas, congresos, redes de } \\
\text { trabajo) }\end{array}$ & 3.14 & .693 & 3.26 & .631 \\
\hline 2.16. Desarrollar procesos de innovación educativa en el aula & 2.93 & .704 & 3.10 & .597 \\
\hline 2.17. Poner en marcha procesos de investigación en el aula & 2.72 & .751 & 2.74 & .729 \\
\hline
\end{tabular}

Tabla 6.

Datos del cuestionario de autopercepción de competencias. Bloque III.

\begin{tabular}{|c|c|c|c|c|}
\hline \multicolumn{5}{|c|}{ Bloque III: Competencias específicas docentes en Educación Física } \\
\hline & \multicolumn{2}{|c|}{$\begin{array}{l}\text { Principio } \\
\text { asignatura }\end{array}$} & \multicolumn{2}{|c|}{$\begin{array}{l}\text { Final } \\
\text { Asignatura }\end{array}$} \\
\hline & Media & DT & Media & DT \\
\hline $\begin{array}{l}\text { 3.1. Diseñar, aplicar y analizar intervenciones didácticas en el área } \\
\text { de Educación Física }\end{array}$ & 2.59 & .733 & 3.23 & .560 \\
\hline $\begin{array}{l}\text { 3.2. Diseñar, desarrollar y evaluar los procesos de enseñanza- } \\
\text { aprendizaje relativos a la actividad física y el deporte con atención } \\
\text { a las Necesidades Específicas de Apoyo Educativo }\end{array}$ & 2.29 & .659 & 3.03 & .669 \\
\hline $\begin{array}{l}\text { 3.3. Conocer y promover las diferentes manifestaciones motrices } \\
\text { que forman parte de la cultura tradicional }\end{array}$ & 2.55 & .736 & 3.03 & .547 \\
\hline $\begin{array}{l}\text { 3.4. Conocer y aplicar los fundamentos biológicos, fisiológicos, de } \\
\text { maduración evolutiva y el desarrollo psicomotor }\end{array}$ & 2.45 & .632 & 3.03 & .657 \\
\hline $\begin{array}{l}\text { 3.5. Diseñar, modificar y/o adaptar al contexto educativo } \\
\text { situaciones motrices orientadas al desarrollo y perfeccionamiento } \\
\text { de las habilidades motrices }\end{array}$ & 2.86 & .639 & 3.29 & .529 \\
\hline $\begin{array}{l}\text { 3.6. Conocer los elementos y fundamentos de la expresión corporal y la } \\
\text { comunicación no verbal y su valor formativo y cultural }\end{array}$ & 2.62 & .862 & 3.26 & .575 \\
\hline $\begin{array}{l}\text { 3.7. Saber utilizar el juego como recurso didáctico y como contenido de } \\
\text { enseñanza }\end{array}$ & 3.59 & .501 & 3.65 & .551 \\
\hline $\begin{array}{l}\text { 3.8. Conocer los fundamentos básicos de la iniciación deportiva escolar y } \\
\text { diseñar tareas específicas para utilizarlos en el ámbito de la enseñanza }\end{array}$ & 2.50 & .745 & 3.10 & .539 \\
\hline $\begin{array}{l}\text { 3.9. Identificar los riesgos que se derivan para la salud de la práctica de } \\
\text { actividades físicas inadecuadas }\end{array}$ & 2.79 & .787 & 2.97 & .752 \\
\hline $\begin{array}{l}\text { 3.10. Diseñar un plan de hábitos de vida saludable (higiene y alimentación) } \\
\text { y de práctica de-actividad física regular. }\end{array}$ & 2.97 & .731 & 3.07 & .691 \\
\hline $\begin{array}{l}\text { 3.11. Saber aplicar los fundamentos (técnicas) de las actividades físicas en } \\
\text { el medio natural }\end{array}$ & 2.76 & .830 & 3.10 & .790 \\
\hline
\end{tabular}


Tabla 6.

Datos del cuestionario de autopercepción de competencias. Bloque III (continuación).

\section{Bloque III: Competencias específicas docentes en Educación Física}

\begin{tabular}{|c|c|c|c|c|}
\hline & \multicolumn{2}{|c|}{$\begin{array}{l}\text { Principio } \\
\text { asignatura }\end{array}$} & \multicolumn{2}{|c|}{$\begin{array}{l}\text { Final } \\
\text { Asignatura }\end{array}$} \\
\hline & Media & DT & Media & DT \\
\hline $\begin{array}{l}\text { 3.12. Saber utilizar diferentes instrumentos de evaluación en el } \\
\text { área de Educación Física }\end{array}$ & 2.82 & .863 & 3.16 & .583 \\
\hline $\begin{array}{l}\text { 3.13. Tener capacidad de reflexión sobre el proceso de } \\
\text { enseñanza/aprendizaje y las distintas metodologías dentro de las } \\
\text { clases de Educación Física }\end{array}$ & 2.83 & .759 & 3.39 & .615 \\
\hline $\begin{array}{l}\text { 3.14. Analizar y comunicar, de manera crítica y fundamentada, el } \\
\text { valor de la actividad física y el deporte y sus posibilidades de } \\
\text { contribuir al desarrollo y bienestar de las personas }\end{array}$ & 3.07 & .716 & 3.29 & .643 \\
\hline
\end{tabular}

\subsection{Rendimiento Académico}

Los resultados globales que se dieron en nuestra experiencia con el alumnado de $3^{\circ}$ de Grado de Educación Infantil fueron los indicados en tabla 7:

Tabla 7.

Distribución del número y porcentaje de alumnos/as según la calificación.

\begin{tabular}{ccc}
\hline & Resultados globales & \\
Calificación & Porcentaje & No alumnos/as \\
\hline Matricula Honor & $4.65 \%$ & 2 \\
Sobresaliente & $9.3 \%$ & 4 \\
Notable & $60.47 \%$ & 26 \\
Aprobado & $4.65 \%$ & 2 \\
Suspenso & $13.95 \%$ & 6 \\
No presentado & $4.98 \%$ & 3 \\
Totales & $\mathbf{1 0 0 \%}$ & $\mathbf{4 3}$ \\
\hline
\end{tabular}

Se puede observar que el rendimiento académico es bastante alto, con casi un $75 \%$ del alumnado con calificación de notable o superior. La tasa de éxito académico también es muy alta, con más de un $80 \%$. Estos resultados vienen a corroborar lo que ya han demostrado otros estudios, que cuando se utilizan sistemas de evaluación formativa y compartida los niveles de éxito académico se incrementan. Por otra parte, es un resultado lógico cuando el alumnado va recibiendo feedback a lo largo de su proceso de aprendizaje.

En la tabla 8 se presenta el \% de alumnado que ha elegido cada una de las tres vías de aprendizaje y evaluación ofertadas. Como puede comprobarse, la más elegida es la vía continua y formativa, seguida de la vía mixta. Muy pocos alumnos eligen la vía examen final.

Tabla 8.

Número de alumnos/as que optan a cada vía.

\begin{tabular}{cccc}
\hline Procesual y formativa & Mixta & Examen & No presentados \\
\hline 67.44 & 23.26 & 2.32 & 6.98 \\
\hline
\end{tabular}

En la tabla 9 se presenta el rendimiento académico en función la vía de aprendizaje y evaluación elegida. Como puede comprobarse, la vía continua es la que tiene un mayor rendimiento académico, con mucha diferencia sobre el resto. Todo el alumnado que ha elegido esta vía obtiene 
calificaciones de notable o superiores, mientras que sólo una cuarta parte de los que eligen la vía mixta son capaces de llegar al notable. Todos los alumnos que optan por el examen final suspenden la asignatura. Este proceso puede considerarse lógico en una asignatura que va trabajando aprendizajes y desarrollo de competencias docentes a lo largo de los cuatro meses. Quién no asiste a clase ni realiza las actividades de aprendizaje programadas es muy difícil que logre desarrollar dichos aprendizajes y competencias.

Tabla 9.

Rendimiento académico entre las distintas vías.

\begin{tabular}{|c|c|c|c|c|c|c|c|}
\hline Vías & NP & Suspenso & $\begin{array}{l}\text { Apro- } \\
\text { bado }\end{array}$ & Notable & Sobresaliente & Mat. Honor & $\begin{array}{c}\text { Totales } \\
\text { vías }\end{array}$ \\
\hline Continua & -- & -- & -- & 53.49 & 9.30 & 4.65 & 67.44 \\
\hline Mixta & & 11.63 & 4.65 & 6.98 & -- & -- & 23.26 \\
\hline Examen & 6.98 & 2.32 & -- & -- & -- & -- & 9.3 \\
\hline $\begin{array}{l}\text { Totales por } \\
\text { calificaciones }\end{array}$ & 6.98 & 13.95 & 4.65 & 60.47 & 9.30 & 4.65 & 100.00 \\
\hline
\end{tabular}

Como resumen de resultados de rendimiento académico, podemos comprobar cómo la mayoría del alumnado superó la asignatura (79,07\%). Siendo la calificación de notable la más común $(60,47 \%)$. La mayoría del alumnado optó por la vía presencial, asistiendo a clase y realizando todas las actividades de aprendizaje (60,47\%). Mientras que un $23.26 \%$ fue por vía Mixta y un porcentaje muy pequeño del alumnado decidió ir por la vía no presencial (2.32\%). En cuanto a la distribución del rendimiento académico podemos observar como el alumnado que realizó la asignatura por la vía presencial obtuvo una calificación mínima de notable, no habiendo ningún suspendo. Por la vía mixta el porcentaje está más repartido en notas más bajas y suspensos, no habiendo ningún alumnado que optase a calificaciones por encima del notable. En cuanto a la vía no presencial, todo el alumnado que se presentó suspendió la asignatura. Podemos afirmar que la obligatoriedad en la continuidad de la asignatura ayuda al alumnado a obtener mejores calificaciones. Mientras que en la vía mixta, donde parte de la calificación depende de ellos, estas notas bajan y el número de suspensos aumenta. Por su parte, el alumnado que se presentó por vía no presencial suspendió al no haber realizado ningún seguimiento de la asignatura hasta el día del examen. Análisis similares pueden encontrarse en Romero et al. (2014).

\subsection{Carga de trabajo para el profesorado y el alumnado}

La carga de trabajo del profesorado se expresa a continuación mediante las tablas 10 y 11.

Tabla 10.

Carga de trabajo para el profesorado.

\begin{tabular}{lcccc}
\hline Asignatura & $\begin{array}{c}\mathbf{N}^{\circ} \text { de } \\
\text { alumnosl } \\
\text { as }\end{array}$ & $\begin{array}{c}\text { número } \\
\text { de grupos }\end{array}$ & $\begin{array}{c}\text { horas } \\
\text { lectivas a la } \\
\text { semana }\end{array}$ & $\begin{array}{c}\text { Horas de } \\
\text { tutorías } \\
\text { semanales }\end{array}$ \\
\hline Fundamentos y didáctica EF en El & 43 & 2 & 6 & 6 \\
\hline
\end{tabular}


Tabla 11.

Carga de trabajo para el profesorado (en horas mensuales).

\section{Asignaturas de un único cuatrimestre}

\begin{tabular}{|c|c|c|c|c|c|c|}
\hline & 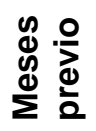 & $\circ \stackrel{\mathscr{g}}{E}$ & $\stackrel{\text { ㅇ }}{E}$ & 욜 & $\stackrel{\circ}{+} \stackrel{g}{E}$ & Totales \\
\hline $\mathrm{PC}=$ preparación de clases & 25 & 14 & 20 & 18 & 12 & 89 \\
\hline$C D=$ corrección de documentos & & 31 & 42 & 41 & 25 & 139 \\
\hline $\begin{array}{l}\mathrm{MW}=\text { mantenimiento de web + e-mail con } \\
\text { alumnado }\end{array}$ & 4 & 6 & 8 & 8 & 7 & 33 \\
\hline Subtotales & 29 & 51 & 70 & 137 & 44 & 261 \\
\hline
\end{tabular}

Como puede apreciarse la carga de trabajo del profesorado es muy grande, especialmente en lo relativo a corrección de documentos, pero también en preparación de clases. La explicación más lógica es que este año la asignatura se ha tenido que impartir por un grupo de 4 profesores noveles, lo cual afecta a esos dos parámetros, tanto porque la preparación de clases existe más tiempo, como porque la menor práctica corrigiendo trabajos y documentos del alumnado hace que necesiten mucho más tiempo para esta actividad. Son dos aspectos en los que la carga de trabajo se rebaja notablemente cuanto mayor es la experiencia docente.

En cuanto a la carga de trabajo del alumnado la analizamos en la tabla 12.

Tabla 12.

Resumen del recuento de horas por meses y actividades de las tareas encomendadas al alumnado.

\begin{tabular}{|c|c|c|c|c|c|c|}
\hline \multicolumn{2}{|c|}{ Tiempo de trabajo que ha supuesto } & $\begin{array}{c}\text { 1er } \\
\text { mes }\end{array}$ & $\begin{array}{c}2^{\circ} \\
\text { mes }\end{array}$ & $\begin{array}{c}3^{\circ} \\
\text { mes }\end{array}$ & $\begin{array}{c}4^{\circ} \\
\text { mes }\end{array}$ & $\begin{array}{l}\text { TOTALES } \\
\text { PARCIALES }\end{array}$ \\
\hline \multicolumn{2}{|l|}{ Lecturas y recensiones. } & & 5 & 10 & 5 & 20 \\
\hline \multicolumn{2}{|c|}{ Preparación y/o informes de sesiones prácticas. } & 8 & 8 & 6 & 6 & 28 \\
\hline \multicolumn{2}{|c|}{ Trabajos e informes de temas. } & 10 & 8 & - & - & 18 \\
\hline \multicolumn{2}{|c|}{$\begin{array}{l}\text { Revisiones y reconducciones de los trabajos (grupal y } \\
\text { en tutoría). }\end{array}$} & 5 & 10 & 5 & & 20 \\
\hline \multicolumn{2}{|l|}{ Estudiar para el examen } & - & & 5 & 12 & 17 \\
\hline \multicolumn{2}{|l|}{ Subtotales por meses } & 23 & 31 & 26 & 23 & 98 \\
\hline \multirow{2}{*}{$\begin{array}{l}\text { Carga media de trabajo } \\
\text { autónomo para el alumnado } \\
\text { a lo largo del cuatrimestre, } \\
\text { aparte de las horas lectivas }\end{array}$} & $\begin{array}{l}\text { Carga del alumno-a que } \\
\text { menos y que más ha }\end{array}$ & \multicolumn{5}{|c|}{$\begin{array}{l}\text { Medias de carga de trabajo (horas) en función de la } \\
\text { calificación del alumnado }\end{array}$} \\
\hline & $\begin{array}{l}\text { hecho en el grupo (en } \\
\text { horas) }\end{array}$ & Suspenso & $\begin{array}{l}\text { Suficie } \\
\text { nte }\end{array}$ & $\begin{array}{l}\text { Notab } \\
\text { le }\end{array}$ & $\begin{array}{l}\text { Sob } \\
\text { salie }\end{array}$ & $\begin{array}{l}\text { Matrícula } \\
\text { Honor }\end{array}$ \\
\hline 98 horas & $\begin{array}{l}\text { valor mínimo: } 47 \text { valor } \\
\text { máximo: } 168\end{array}$ & 50 & 69 & 90 & 14 & 150 \\
\hline
\end{tabular}


La carga media de trabajo del alumnado es de 98 horas, por lo que se ajusta muy bien a la carga no lectiva prevista (90 horas). Es ligeramente superior, pero no tanto como siempre considera el alumno. Respecto a la distribución de la carga de trabajo según las calificaciones obtenidas, podemos comprobar cómo el alumnado con calificación de notable se ajusta muy bien a la carga prevista; en cambio, las notas más altas, sobresaliente y matrícula de honor, afirma haber hecho un número de mayor que la carga lectiva prevista horas (entre 140-150 horas totales, respecto a las 90 no presenciales previstas). Mientras que el alumnado con notas suspensas o de suficiente afirma estar lejos de la carga de 90 horas, lo cual también ayuda a explicar la baja calificación; si no realiza todo el trabajo previsto en la asignatura, es complicado que adquiera los conocimientos y competencias planificados.

\section{CONCLUSIONES}

En general la experiencia ha sido muy positiva. La idea es mantener la práctica de éxito y volver a utilizar de forma sistemática la ficha detallada de autoevaluación y autocalificación (escala descriptiva, elaborada por López-Pastor et al., 2016).

Como puede apreciarse la carga de trabajo del profesorado se ha incrementado notablemente respecto a otros años. La única explicación que encontramos es que este año la asignatura se ha tenido que impartir por un grupo de 4 profesores noveles, lo cual afecta a esos parámetros, tanto porque la preparación de clases existe más tiempo, como porque la menor práctica corrigiendo trabajos y documentos del alumnado hace que necesiten mucho más tiempo para esta actividad. Son dos aspectos en los que la carga de trabajo se suele rebajar notablemente cuanto mayor es la experiencia docente.

\section{REFERENCIAS}

Barba, J. J., López Pastor, VM., Manrique Arribas, JC., Gea Fernández, JM., \& Monjas Aguado, R. (2010). Garantir l'èxit en la formació inicial del professorat d'educació física: els projectes d'aprenentatge tutelats. Temps d'educació, 39, 187-206.

Barba-Martín, R.A., \& López Pastor, VM. (2017). Evaluación formativa y compartida en los proyectos de trabajo tutorado, un ejemplo de buena práctica. Revista Infancia, Educación y Aprendizaje 3(2), 66-70.

Biggs, J. (2005). Calidad del aprendizaje universitario. Madrid. Narcea.

Brown, S., \& Glasner, A. (2003). Evaluar en la Universidad. Problemas y nuevos enfoques. Madrid: Narcea.

Hamodi Galán, C., Moreno-Murcia, J. A., \& Barba-Martín, R.A. (2018). Medios de evaluación y desarrollo de competencias en Educación Superior en estudiantes de Educación Física. Revista de Estudios Pedagógicos, 44(2), 241-257.

López, V. M. (Coord.) (2006). La Evaluación en Educación Física: revisión de los modelos tradicionales y planteamiento de una alternativa: la evaluación formativa y compartida. Buenos Aires: Miño y Dávila.

López, V.M., Castejón, J., \& Pérez, Á. (2012). ¿Implicar al alumnado en la evaluación en la formación inicial del profesorado? Un estudio de caso de evaluación entre iguales de un examen. Multidisciplinary Journal of Educational Research, 2(2), 177-201. doi: 10.4471/remie.2012.09.

López-Pastor, V.M., Manrique Arribas, J. C., Monjas Aguado, R., \& Gea Fernández, J. M. (2010). Formative Assessment in project-oriented learning to improve academic performance. Assessment, Learning \& Teaching Journal, 9, 23-26.

López Pastor,V. M., Monjas Aguado, R., Manrique Arribas, J. C., Barba, J. J., \& González Pascual, M. (2008). Implicaciones de la evaluación en los enfoques de educación física cooperativa: El papel de la evaluación formativa y compartida en la necesaria búsqueda de coherencia. C \& E: Cultura y Educación, 20(4), 457-477. 
López-Pastor, V. M., Pérez-Pueyo, A., Barba, J. J., \& Lorente, E. (2016). Rubricas y PATS. Utilización de una escala graduada (rubrica) para la autoevaluación de trabajos escritos en la formación inicial del profesorado de educación física (FIPEF). Percepción de su funcionalidad por parte de los estudiantes. Cultura, Ciencia, Deporte (CCD), 31(11,1), 37 49. DOI: $10.12800 / \mathrm{ccd}$.

Manrique Arribas, J.C., López Pastor, V.M., Monjas Aguado, R., \& Real Rubio, F. (2010). El potencial de los proyectos de aprendizaje tutorado y los sistemas de evaluación formativa en la mejora de la autonomía del alumnado. Una experiencia interdisciplinar en formación inicial del profesorado. Revista Española de Educación Física y Deportes, 14, 39-57.

Romero-Martín, R., Fraile-Aranda, A., López-Pastor, V. M., \& Castejón-Oliva, F. J. (2014). Relación entre sistemas de evaluación formativa, rendimiento académico y carga de trabajo del profesor y del alumno en la docencia universitaria. Revista Infancia y Aprendizaje, 37(1), 1632. 
ANEXO I-HOJA DE INFORMACIÓN PARA EL ALUMNADO SOBRE PROCESO REALIZACIÓN PROYECTO DE APRENDIZAJE TUTORADO (PAT)

\begin{tabular}{|c|c|c|}
\hline NOMBRE: & \multicolumn{2}{|l|}{ PROYECTO DE APRENDIZAJE TUTORADO } \\
\hline \multicolumn{3}{|c|}{$\begin{array}{l}\text { NOMBRE Y DATOS DEL ALUMNOIA: } \\
\text { ASIGNATURA Y CURSO }\end{array}$} \\
\hline \multicolumn{2}{|c|}{$\begin{array}{l}\text { CARÁCTER DE LA PRÁCTICA: Llevar a cabo un proyecto de aprendizaje tutorado grupal, } \\
\text { sobre una temática propuesta. } \\
\text { ES COLECTIVO. Grupo entre } 3 \text { y } 5 \text { personas. Preferentemente el mismo grupo que en las } \\
\text { otras asignaturas de la mención. }\end{array}$} & FECHA DE REALIZACIÓN: \\
\hline \multicolumn{3}{|c|}{$\begin{array}{l}\text { RELACIÓN CON LOS CONTENIDOS DEL PROGRAMA: } \\
\text { Bloque de contenidos }\end{array}$} \\
\hline \multicolumn{3}{|c|}{$\begin{array}{l}\text { A-MARCO TEÓRICO DEL PAT. (máximo de cuatro hojas con al menos cuatro lecturas relacionadas entre sí). } \\
\text { B-PLAN DE SESIÓN: 1-Eje; 2-contenido principal; 3-metodología (directiva / no directiva, tipo de tareas y estilos de enseñanza); } \\
\text { 4-Evaluación (diferenciando entre técnicas e instrumentos, mejor utilizar tabla); 5-roles de los profesores/as }\end{array}$} \\
\hline $\begin{array}{l}\text { ESTRUCTU } \\
\text {-datos y títu } \\
\text { 1-PLAN (Ej } \\
\text { tareas y est } \\
\text { diversidad ( } \\
\text { + plan deta } \\
\text { 2-NARRAD } \\
\text { 3-ANÁLISIS } \\
\text { 4-EVALUAC } \\
\text { aprendizaje }\end{array}$ & $\begin{array}{l}\text { QUE DEBE TENER EL INFORME FINAL A REALIZAR } \\
\text { Informe del PAT...." } \\
\text { ontenido principal; contenidos secundarios (justificar donde se dan), metodolc } \\
\text { de enseñanza); Evaluación (diferenciando entre técnicas e instrumentos, mej } \\
\text { enciando entre la diversidad natural del grupo-aula y la atención a los alumnd } \\
\text { o de la sesión a realizar, con los diálogos incluidos. } \\
\text { ora y minuto, narrado detallado grupo, narrado detallado maestras/os). } \\
\text { MPETENCIAS DOCENTES: las habituales, ver sesión. } \\
\text { J DEL PAT. (A-variaciones respecto a lo planificado; B-revisión del proceso s } \\
\text { oración general, etc.). }\end{array}$ & $\begin{array}{l}\text { (directiva / no directiva, tipo de } \\
\text { tilizar tabla); atención a la } \\
\text { on NEE; roles de las profesoras/es } \\
\text { uido, problemas, soluciones, }\end{array}$ \\
\hline \multicolumn{3}{|c|}{$\begin{array}{l}\text { 1-Realizar el marco teórico y el plan de sesión y llevarlos a la tutoría correspondiente. Como } \\
\text { la sesión con los compañeros. }\end{array}$} \\
\hline \multicolumn{3}{|c|}{ 2-Corregir lo realizado con el profesor en el seminario y/o tutorías. } \\
\hline \multicolumn{3}{|c|}{$\begin{array}{l}\text { 3-Corregir lo señalado por el profesor y volver a revisarlo con él. Este proceso se repite hasta que el profesor dé el visto bueno } \\
\text { definitivo para llevarlo a cabo. Hacer } 60 \text { copias del marco teórico. } \\
\text { 4-Llevar a cabo la sesión con los compañeros + repartir el marco teórico + explicárselo a los compañeros + análisis colectivo de la } \\
\text { sesión. } \\
\text { 5-Realizar el informe. Plazo máximo de entrega: una semana después de haber realizado la sesión. }\end{array}$} \\
\hline \multicolumn{2}{|c|}{ POSIBLES PAT A ELEGIR. Seleccionar uno de los siguientes PAT y comunicárselo al profe } & -Ambientes de Aprendizaje (2) \\
\hline \multicolumn{3}{|c|}{-Espacios de Acción y Aventura (2) } \\
\hline \multicolumn{3}{|c|}{-Tratamiento pedagógico de lo corporal (2) } \\
\hline \multicolumn{3}{|c|}{-Estilos De Enseñanza (1) } \\
\hline \multicolumn{3}{|c|}{-EF En Centros Bilingües (2) } \\
\hline \multicolumn{3}{|c|}{-Pick y Vayer (1) } \\
\hline \multicolumn{3}{|c|}{-Lapierre y Aucouturier (1) } \\
\hline \multicolumn{3}{|c|}{-Le Boulch (1) } \\
\hline \multicolumn{3}{|c|}{-Viscarro y Camps (1) } \\
\hline \multicolumn{3}{|c|}{-Cuñas motrices (1-2) } \\
\hline
\end{tabular}


ANEXO II - FICHA PARA LA EVALUACIÓN DEL PROYECTO DE APRENDIZAJE TUTORADO

\begin{tabular}{|l|l|}
\hline \multicolumn{1}{|c|}{ NOMBRE DEL P.A.T.: } \\
\hline $\begin{array}{l}\text { Posibles aspectos a } \\
\text { evaluar }\end{array}$ & \\
\hline $\begin{array}{l}\text { Diferencias entre lo } \\
\text { planificado y lo ocurrido }\end{array}$ & \\
\hline $\begin{array}{l}\text { Reuniones internas } \\
\text { llevadas a cabo y } \\
\text { asistencia }\end{array}$ & \\
\hline $\begin{array}{l}\text { Reuniones con el } \\
\text { profesor y breve } \\
\text { comentario de lo } \\
\text { realizado }\end{array}$ & \\
\hline $\begin{array}{l}\text { Problemas que surgen } \\
\text { durante el proceso y } \\
\text { como se solucionan }\end{array}$ & \\
\hline $\begin{array}{l}\text { Valoración del proceso } \\
\text { de aprendizaje que se } \\
\text { lleva a cabo a lo largo } \\
\text { del PAT }\end{array}$ & \\
\hline $\begin{array}{l}\text { Otros aspectos a } \\
\text { comentar }\end{array}$ & \\
\hline
\end{tabular}


ANEXO III - FICHA DE AUTOEVALUACIÓN GRUPAL (Proyecto de Trabajo tutorado...):

\begin{tabular}{|c|c|c|c|c|c|c|c|c|c|}
\hline \multicolumn{2}{|c|}{$\begin{array}{l}\text { Componentes Del Grupo: } \\
\text { 1-Coordinador-a: } \\
\text { 2-Secretario-a: }\end{array}$} & \multicolumn{7}{|c|}{$\begin{array}{l}\text { 3-Vocal: } \\
\text { 4-Vocal: } \\
\text { 5-Vocal: }\end{array}$} & \multirow{3}{*}{\begin{tabular}{|l}
$\begin{array}{l}\text { Fecha de entrega: } \\
\text { Tipo de trabajo: }\end{array}$ \\
Observaciones
\end{tabular}} \\
\hline \multirow{2}{*}{\multicolumn{2}{|c|}{ Aspectos a evaluar }} & \multicolumn{6}{|c|}{$\begin{array}{l}\text { COMPONENTES DEL } \\
\text { GRUPO }\end{array}$} & \multirow[t]{2}{*}{$\begin{array}{l}\text { Calidad del } \\
\text { documento }\end{array}$} & \\
\hline & & 1 & 2 & 3 & 4 & 5 & 6 & & \\
\hline \multicolumn{2}{|l|}{ Aprendizaje } & & & & & & & & \\
\hline \multicolumn{2}{|c|}{ Organización y presentación } & & & & & & & & \\
\hline \multicolumn{2}{|c|}{ Utilización bibliografía y citas } & & & & & & & & \\
\hline \multicolumn{2}{|c|}{ Desarrollo del contenido } & & & & & & & & \\
\hline \multicolumn{2}{|c|}{$\begin{array}{l}\text { Aportaciones propias - calidad del } \\
\text { análisis }\end{array}$} & & & & & & & & \\
\hline \multicolumn{2}{|c|}{$\begin{array}{l}\text { - Tiempo de elaboración (en } \\
\text { minutos) }\end{array}$} & & & & & & & & \\
\hline \multicolumn{2}{|l|}{ Otros aspectos: } & & & & & & & & \\
\hline \multicolumn{2}{|c|}{$\begin{array}{l}\text { Aportación de cada uno al trabajo } \\
\text { grupal }\end{array}$} & & & & & & & & \\
\hline \multicolumn{10}{|c|}{ Acta de reuniones: } \\
\hline NIVEL A & \multicolumn{3}{|l|}{ NIVEL B } & \multicolumn{5}{|c|}{ NIVEL C } & NIVEL D \\
\hline $\begin{array}{l}\text { 1-Marco teórico } \\
\text { muy bien } \\
\text { elaborado. } \\
\text { 2-Alto dominio de } \\
\text { las competencias } \\
\text { docentes durante } \\
\text { el desarrollo de la } \\
\text { sesión práctica; } \\
\text { 3-Muy buena } \\
\text { presentación oral } \\
\text { del marco teórico } \\
\text { (clara, sin leer, } \\
\text { mirar a todos, etc.). } \\
\text { 4-Informe final bien } \\
\text { presentado y } \\
\text { elaborado y buen } \\
\text { análisis de la } \\
\text { sesión }\end{array}$ & \multicolumn{3}{|c|}{$\begin{array}{l}\text { 1-Marco teórico bien } \\
\text { elaborado. } \\
\text { 2-Buen dominio de las } \\
\text { competencias docentes } \\
\text { durante el desarrollo de } \\
\text { la sesión práctica; } \\
\text { 3-Buena presentación } \\
\text { oral del marco teórico } \\
\text { (clara, sin leer, mirar a } \\
\text { todos, etc.). } \\
\text { 4-Informe final bien } \\
\text { presentado y elaborado } \\
\text { y buen análisis de la } \\
\text { sesión }\end{array}$} & \multicolumn{5}{|c|}{\begin{tabular}{|l} 
1-Marco teórico bien \\
elaborado. \\
2-Algunos problemas en \\
las competencias docentes \\
durante el desarrollo de la \\
sesión práctica; \\
3-Algunos problemas en la \\
presentación oral del \\
marco teórico (poco clara, \\
leer, no mirar a todos, \\
etc.). \\
4-Informe final bien \\
presentado, pero un \\
análisis demasiado simple \\
o erróneo de lo sucedido \\
en la sesión
\end{tabular}} & $\begin{array}{l}\text { 1-Marco teórico mal } \\
\text { elaborado, incompleto o mal } \\
\text { presentado. } \\
\text { 2-Problemas serios en las } \\
\text { competencias docentes } \\
\text { durante el desarrollo de la } \\
\text { sesión práctica. } \\
\text { 3-Mala presentación oral del } \\
\text { marco teórico (poco clara, } \\
\text { leer, sin preparar, etc.). } \\
\text { 4-Informe final mal } \\
\text { presentado, faltan apartados, } \\
\text { análisis demasiado simple o } \\
\text { erróneo de lo sucedido en la } \\
\text { sesión. }\end{array}$ \\
\hline
\end{tabular}

